L'HOMME L'Homme

Revue française d'anthropologie

$201 \mid 2012$

Varia

\title{
Écritures ordinaires en pays touareg
}

\section{Dominique Casajus}

\section{OpenEdition}

Journals

Édition électronique

URL : http://journals.openedition.org/lhomme/22929

DOl : $10.4000 /$ lhomme.22929

ISSN : 1953-8103

\section{Éditeur}

Éditions de l'EHESS

\section{Édition imprimée}

Date de publication : 23 février 2012

Pagination : 31-54

ISSN : 0439-4216

Référence électronique

Dominique Casajus, «Écritures ordinaires en pays touareg », L'Homme [En ligne], 201 | 2012, mis en ligne le 15 février 2014, consulté le 01 mai 2019. URL : http://journals.openedition.org//homme/22929 ; DOI : 10.4000/lhomme.22929

(c) École des hautes études en sciences sociales 


\title{
Écritures ordinaires en pays touareg
}

\author{
Dominique Casajus
}

S

ELON LES CATÉGORISATIONS qu'ils affectionnent, les ethnologues rangeraient plutôt la société touarègue parmi les sociétés de culture orale. Les choses sont en réalité plus complexes, car s'il est un fait que leur littérature est presque exclusivement orale, les Touaregs écrivent, et depuis fort longtemps. Ils disposent pour cela d'un vieil alphabet dont les caractères reçoivent le nom spécifique de tafineq (pl. tifinagh), mot qui, au pluriel, sert à désigner l'alphabet lui-même. Cet alphabet autochtone coexiste avec deux alphabets d'origine étrangère : l'alphabet arabe, que l'islam a introduit chez eux il y a plusieurs siècles, et l'alphabet latin, que la scolarisation commence à répandre. Ceux-ci ne reçoivent pas de dénomination particulière : on leur applique un terme générique dont les réalisations dialectales sont esekkil ou eshekkul (pl. isekkilen ou ishekkulen), et qui désigne tout signe graphique, à quelque système d'écriture qu'il appartienne. Presque tous les Touaregs ont une connaissance au moins rudimentaire de leur propre alphabet, alors que seuls ceux, relativement peu nombreux encore, qui ont fréquenté l'école coranique ou l'enseignement officiel maîtrisent les alphabets arabe ou latin. Je parlerai ici surtout du premier, tout en faisant leur place aux deux autres car leur présence à ses côtés n'est pas sans incidence sur la perception qu'en ont ses utilisateurs. Une perception sur laquelle pèse aussi le passé qu'ils lui supposent. C'est pourquoi je commencerai par évoquer ce passé, avant d'aborder ce qui constitue le principal usage

Une première version de cet article a été exposée au colloque "Anthropologie et histoire de la mutation graphique et de ses effets. Autour d'Armando Petrucci " (Rome, janvier 2003). 
de cet alphabet : l'art épistolaire. Le présent article se veut donc à la fois une contribution à l'ethnographie d'une société particulière et une intervention dans les débats que plusieurs parmi nous consacrent aux écritures qu'ils appellent quotidiennes, ordinaires ${ }^{1}$, ou encore domestiques ${ }^{2}$.

\section{Un vieil alphabet}

Pour les Touaregs sahéliens, les inscriptions rupestres qu'on rencontre sur leurs terres de parcours, parfois si vieilles qu'ils ne parviennent pas à les déchiffrer, attestent la grande antiquité de leur écriture. Ils attribuent les plus anciennes à un personnage nommé, selon les régions, Aniguran ou Arigulan, qui les aurait tracées de son doigt aux temps lointains où il pleuvait tant sur le monde que les rochers s'étaient amollis. Quant à l'alphabet lui-même, une légende reçue à l'ouest du Niger en fait remonter l'origine à l'antéislam et donne à son inventeur le nom d'Amerolqis (Aghali-Zakara \& Drouin 1979, 2007), vocable sous lequel on reconnaît une figure illustre de l'Arabie archaïque: le poète Imrûl-Qays. C'est que, comme l'étude des poésies antéislamiques a été tenue depuis l'époque omayyade pour indispensable à la bonne compréhension du Coran, elles se sont diffusées très tôt jusqu'aux extrémités du monde musulman, avec la geste et le nom de leurs auteurs supposés (Casajus 2000a). Tel est pour les Touaregs le paradoxe de leur alphabet: le nom du héros païen auquel ils attribuent son invention leur a été enseigné par ceux-là mêmes qui, en les convertissant à l'islam, ont répandu parmi eux l'usage d'un autre alphabet. Il nous sera décidément difficile d'oublier que les tifinagh coexistent avec d'autres isekkilen.

Plus prosaïques qu'eux, nos érudits s'accordent cependant avec les Touaregs sur l'ancienneté des tifinagh. Dès le milieu du XIX siècle, ils les ont rapprochées de l'écriture dite libyque, connue par des inscriptions découvertes dans les régions où les Anciens situaient la Numidie et la Mauritanie. Certaines de ces inscriptions sont des bilingues où l'alphabet libyque côtoie le punique ou le latin, ce qui a permis le déchiffrement au moins partiel de ses variantes les plus orientales. Leur langue elle-même nous est largement inconnue mais quelques mots déchiffrés laissent penser qu'elle se rattache à la famille berbère, dont le touareg fait partie. Aucune de ces inscriptions n'a pu être datée avec certitude, hormis une bilingue mise au jour à Dougga (Tunisie), et que son incipit situe « en l'an

1. Je pense notamment aux volumes édités par Daniel Fabre (1993, 1997), ainsi qu'aux travaux de Bernard Lahire (cf., notamment, $2001: 211$ sq.).

2. Pour reprendre le titre de la contribution de Jean-Pierre Albert à l'un des deux volumes cités précédemment (Albert 1993). 
dix du [roi] Micipsa", c'est-à-dire en 139 avant J.-C ${ }^{3}$. Plusieurs signes libyques se retrouvent parmi les tifinagh avec la même valeur et des formes semblables. Ces similitudes autorisent à supposer que les alphabets libyques ont évolué vers les tifinagh modernes, dans une dérive bimillénaire dont des inscriptions sahariennes encore obscures représenteraient les états intermédiaires, et les gravures sahéliennes attribuées à Aniguran, le stade pénultième.

L'origine de l'alphabet libyque a fait l'objet d'un long débat. Certains y ont vu un emprunt au punique. De fait, quelques signes libyques ressemblent à leurs correspondants dans les alphabets sémitiques anciens; à quoi s'ajoute la proximité, relevée depuis longtemps, entre tafineq, où l'on peut isoler une racine FNGh ou FYNGh ${ }^{4}$, et le mot avec lequel les Grecs désignaient les Phéniciens. Si tafineq remonte à l'Antiquité, il faudrait supposer que les anciens Libyques, eux aussi, disaient écrire avec des phoinikeia grammata. L'hypothèse de l'emprunt au punique s'exprime cependant aujourd'hui avec quelques nuances: s'il ne fait pas de doute que les peuples auxquels nous devons les inscriptions dites libyques ont emprunté aux colons puniques l'idée de l'écriture alphabétique en tant que telle, les signes en eux-mêmes semblent avoir été pour une bonne part une création locale (Février 1959: 323 ; Galand 2001: 2 ; Pichler 2007).

Aujourd'hui, les Touaregs n'utilisent les tifinagh que pour des textes courts : lettres à des proches, graffiti sur les arbres, sur les rochers ou les ustensiles quotidiens. On écrit de droite à gauche, de gauche à droite, de bas en haut, ou en boustrophédon. En principe, seules les consonnes sont notées. Cependant, certains scripteurs notent sporadiquement les voyelles en fin de mot par un point. Au Hoggar, ce point note seulement le $a$, tandis que le $u$ ou le $i$ finaux sont notés respectivement par W et $\mathrm{Y}$, usage qui rappelle les matres lectionis des anciens Sémites et dont nous allons voir qu'il inspire aujourd'hui les créateurs d'alphabet. Deux consonnes qui se suivent sans intervalle vocalique peuvent être figurées par une ligature. Par exemple, on peut figurer la séquence consonantique $r t$ par le signe $\oplus$, où le + notant le $t$ est inscrit dans le $\mathrm{O}$ notant le $r$. Ces ligatures n'existent pas pour toutes les séquences susceptibles d'apparaître dans la langue, et seuls les scripteurs les plus expérimentés savent les utiliser. Les signes se suivent le plus souvent sans discontinuité, mais quelques scripteurs ont l'habitude d'isoler les mots par un trait [voir planches].

3. Gabriel Camps a cru pouvoir faire remonter au VI siècle avant J.-C. une inscription trouvée dans l'Atlas marocain, mais, comme je l'ai montré ailleurs (Casajus 2011), ses arguments, repris un peu hâtivement par Chaker \& Hachi (2000), étaient fragiles.

4. Le $G h$ (ou $g h)$ note ici la vélaire constrictive sonore. 
La lecture se fait toujours à haute voix. Pour emprunter un exemple à une des lettres qui seront commentées plus loin, le lecteur ayant à déchiffrer la séquence RGhLFSNSN chante une sorte de récitatif qu'on peut transcrire comme RäGhäL $\ddot{a} F \ddot{a} S \ddot{a} N \ddot{a} S \ddot{a} N \ddot{a}$, où $\ddot{a}$ transcrit une voyelle d'articulation à peu près centrale; et il interprète cette séquence sonore comme eRiGh eLFaSeN eSsiN: "je veux/pioches/deux (= je voudrais deux pioches)». La lecture suppose donc un passage par l'oral. Non pas seulement parce qu'on lit toujours à haute voix ; ni non plus parce que, comme pour un texte hébraïque ou arabe dépourvu de signes diacritiques, l'absence des voyelles réclame qu'un lecteur vienne les apporter pour rendre le texte compréhensible (Alvarez-Pereyre 1989: 25). Car l'hébraïsant ou l'Arabe qui prononce les voyelles brèves absentes de l'écrit profere une parole immédiatement intelligible. Ce que le Touareg prononce n'est pas un texte où il aurait incorporé les voyelles manquant dans la graphie, mais une séquence sans signification où les voyelles ont une valeur indéterminée. Entre le LFSNSN qu'il a sous les yeux et le $e L F a S e N$ eSsiN qu'il comprend finalement, s'intercale le $L \ddot{a} F \ddot{a} S \ddot{a} N \ddot{a} S \ddot{a} N \ddot{a}$ qu'il a prononcé à haute voix, intermédiaire qui n'existe pas pour le lecteur hébreu ou arabe. Celui-ci, comme d'ailleurs un lecteur français qui pratiquerait la lecture à haute voix, s'entend lire ce qu'il déchiffre; le Touareg déchiffre ce qu'il s'entend lire. La lecture est pour lui la combinaison de deux opérations disjointes: l'émission d'une séquence sonore, et un déchiffrement. Opérations qui peuvent d'ailleurs être effectuées par deux personnes distinctes: lorsqu'un lecteur syllabe à haute voix, ceux qui l'entourent déchiffrent en même temps que lui, en se fondant sur ce qu'ils entendent et non sur ce qu'ils lisent. C'est d'ailleurs souvent comme cela que procédaient les Touaregs qui me demandaient de leur lire leurs lettres. Je devais lire à leur manière, tandis qu'ils déchiffraient la suite de sons que j'émettais à haute voix sans toujours comprendre. Bien entendu, lorsque le lecteur devine d'emblée le sens de ce qu'il a sous les yeux, il peut se dispenser de la syllabation intermédiaire, mais il est forcé d'y revenir dès que ce sens n'est pas immédiatement apparent.

Voilà qui justifie une brève digression. Ignace Gelb (1973) proposa naguère de voir dans les anciennes écritures sémitiques non des alphabets consonantiques comme on le fait généralement, mais des syllabaires où le signe $C$ représenterait une syllabe $C x$ composée d'une consonne donnée $c$ et d'une voyelle variable $x$ que seul le contexte détermine. Ses arguments étaient forcément indirects. Ainsi, le fait que les anciens Sémites aient éprouvé le besoin de créer des marques comme le sukun arabe ou le shewa hébreu pour indiquer le manque de voyelle signifiait à ses yeux que «chaque signe valait d'abord pour une syllabe complète, c'est-à-dire une 
syllabe [il voulait dire "consonne"] plus une voyelle" (ibid.: 164). De la même manière, le lecteur touareg prononce une suite de syllabes dont les consonnes ont une valeur précise et les voyelles une valeur arbitraire qu'il ne détermine qu'après coup. On serait tenté d'en déduire que les tifinagh sont un syllabaire si l'argumentation de Gelb ne montrait aussi, incidemment, que la frontière est floue entre syllabaire et alphabet consonantique. Considérons, en effet, les ligatures. Quoique de façon moins systématique, elles jouent en touareg le même rôle que le sukun en arabe. Pour noter la séquence $r t$ par exemple, le Touareg use du signe $\oplus$, là où l'Arabe placerait un sukun entre le $r$ et le $t$. Mettons, pour parler comme Gelb, que ce signe note la syllabe $R T \ddot{a}$. Il faut alors remarquer, puisque le $\oplus$ combine le $\mathrm{O}(r)$ et le $+(t)$, que le Touareg qui l'utilise perçoit dans la syllabe $R T \ddot{a}$ des éléments phoniques présents dans les syllabes $R \ddot{a}$ et $T \ddot{a}$. Or, si $T \ddot{a}$ se retrouve effectivement dans $R T \ddot{a}$, le seul élément de R $\ddot{a}$ qui s'y conserve est la consonne $R$. L'écriture touarègue isole donc indiscutablement les consonnes, au moins lorsqu'un contexte phonique particulier permet de les percevoir indépendamment de la syllabe dont elles font partie ; il en va de même pour les voyelles, dont on a vu qu'elles sont occasionnellement notées en fin de mot, c'est-à-dire lorsque, la voix s'arrêtant sur elles, il devient possible de les percevoir isolément. En un mot, si la lecture des tifinagh est assurément une syllabation, le syllabaire que Gelb y aurait peut-être vu porte en lui un alphabet, souvent invisible, quelquefois visible. Autant dire qu'elles sont tout de même d'une nature un peu ambiguë.

En tout cas, les Touaregs ont bien conscience qu'ils ne lisent pas leur écriture comme leurs clercs lisent l'arabe. La lecture des tifinagh est désignée par le verbe ennen, qui pourrait bien être un idéophone formé à partir de ennu, "dire", par redoublement de la racine (mode fréquent de formation des idéophones en touareg). Voici ce que Foucauld (19511952 : III, 1391-1392) écrit sous la rubrique ennen dans son Dictionnaire touareg-français :

" [...] épeler (lire en épelant) [...] peut avoir pour rég[ime] dir[ect] n’importe quel écrit, n'importe quels caractères (appartenant à un alphabet et à une langue quelconques). Signifie épeler n'importe comment, intérieurement et sans mouvement de lèvres, à voix basse, à mi-voix, ou à haute voix. Differe d'egher, "lire" (n'importe quelle écriture, n'importe quel écrit, n'importe quels caractères appartenant à un alphabet et à une langue quelconques [...] intérieurement et sans mouvement de lèvres, ou à voix basse, ou à mi-voix, ou à haute voix). Comme il est rare, par suite du manque de voyelles, de séparation entre les mots et de ponctuation, qu'on puisse lire l'écriture touarègue autrement qu'en épelant lentement et en tâtonnant, c'est habituellement le verbe ennen, et non le verbe egher, qui est employé pour exprimer la lecture en caractères touaregs ». 
En réalité, lorsqu'il est appliqué à la lecture en tifinagh, ennen ne désigne pas exactement une épellation, mais la syllabation décrite plus haut. Et le verbe egher, dont l'étymon arabe a donné notre Qoran, désigne en premier lieu la lecture psalmodiée du texte coranique.

Le vocabulaire relatif à l'écriture montre également que les Touaregs perçoivent les diverses sortes d'écrits comme étant de nature différente. Si l'acte d'écrire est rendu de façon générale par le verbe d'origine arabe ekteb, il n'existe aucun terme générique pour en désigner le résultat, l'écrit. Elkettab, mot dérivé de ekteb que certains auteurs prétendent traduire par «livre", s'applique avant tout aux textes rédigés en caractères arabes. Pour désigner un livre écrit en caractères latins, on préfere utiliser les mots d'origine française libr ou cayé. Tirot, terme plus compréhensif puisqu'il peut s'appliquer à un texte utilisant n'importe quel alphabet, ne désigne que des pièces assez courtes pour tenir sur quelques feuilles de papier, comme les lettres, les amulettes coraniques, les documents administratifs (Galand 1976 ; Casajus 2000b : chap. VIII).

Des intellectuels berbérophones, kabyles ou marocains, ont entrepris depuis quelques décennies de donner aux tifinagh un statut comparable à celui des alphabets arabe ou latin. À des consonnes inspirées des tifinagh ou du libyque, ils ont ajouté, en généralisant l'usage que l'écriture touarègue réserve aux syllabes finales, les voyelles $a$ (notée par un point), $u$ et $i$ (notées par le W et le Y touaregs), ainsi que le shewa kabyle, pour lequel ils ont forgé un signe particulier. Quelques Touaregs ont tenté à leur tour d'adapter les tifinagh à une écriture cursive et de leur adjoindre des voyelles. Ces initiatives font l'objet d'un débat dans lequel je me garderai d'intervenir, sauf pour faire remarquer qu'elles aboutissent à la création d'alphabets illisibles pour les Touaregs non scolarisés. Là est, si je comprends bien, la raison pour laquelle le linguiste touareg Mohamed Aghali-Zakara (1994) les a sévèrement critiquées : à quoi bon moderniser un système d'écriture si c'est pour le rendre inaccessible à ceux qui l'utilisent fort bien tel qu'il est? Ceux-ci, en effet, ne peuvent appliquer à cette écriture nouvelle la syllabation à laquelle ils sont habitués, et doivent pour y accéder s'imposer le même apprentissage que ceux qui sont passés par l'école. Pour le dire d'un mot, les créateurs d'alphabets ont manqué à percevoir la différence qualitative qui sépare les deux modes de lecture que les Touaregs appellent respectivement ennen et egher. Habitués à la lecture du français ou de l'arabe, ils ont forgé à partir d'une écriture adaptée à la syllabation un instrument qui ne s'y prête plus.

Signalons par ailleurs que les Touaregs de la boucle du Niger, dont certains parlent aussi bien l'arabe que leur langue maternelle, ont, depuis au moins les années 1960, l'habitude d'adjoindre aux tifinagh le sukun 
ainsi que les signes diacritiques dont l'arabe note les voyelles brèves. Les textes ainsi produits restent accessibles à un Touareg non scolarisé, puisque les signes ajoutés se placent sans la modifier en haut ou en bas de la ligne d'écriture à laquelle il est habitué (Coninck \& Galand 1960).

On voit donc que les tentatives actuelles pour adjoindre des voyelles aux alphabets traditionnels s'inspirent de l'arabe ou du kabyle, peut-être aussi du français. On mesure ici combien les tifinagh n'existent que dans le voisinage d'autres alphabets - et même d'autres langues. On mesure aussi que ce ne sont pas seulement des alphabets différents qui se côtoient, mais aussi des modes de lecture différents. Je reparlerai plus loin de l'utilisation occasionnelle du français, mais disons déjà qu'on trouve dans certaines régions des clercs qui maîtrisent suffisamment l'arabe pour pouvoir transcrire du touareg en utilisant l'alphabet arabe. Je n'ai personnellement jamais vu de textes ainsi transcrits, la maîtrise de l'arabe étant peu répandue chez les Touaregs auprès desquels j'ai séjourné. La seule utilisation de l'alphabet arabe que je puisse rapporter est celle-ci : mon ami et collaborateur Moussa Albaka y recourt pour remplir son répertoire téléphonique, en notant systématiquement les voyelles comme longues. Sans doute parce qu'il a constaté que les tifinagh sont mal adaptées à la transcription de noms étrangers où les voyelles ont plus d'importance qu'en touareg.

\section{Les lettres au marabout}

Venons-en maintenant à l'emploi des tifinagh dans l'art épistolaire. J'utiliserai deux corpus, datant respectivement de 1913-1914 et de la fin des années 1880 : des lettres écrites à Charles de Foucauld par ses amis du Hoggar; des lettres que des Touaregs du Sahara central, emprisonnés à Alger pour avoir razzié des campements arabes, ont échangées avec leurs familles. Quoique anciens, ces deux corpus sont très comparables à ceux qu'on pourrait constituer aujourd'hui. Citons pour commencer une lettre écrite en mai 1913, alors que Foucauld voyageait en France avec Ouksem, un adolescent de la tribu roturière des Dag-Ghali ${ }^{5}$. Chikat et Choumekki $\mathrm{y}$ sont le père et la mère du jeune voyageur; Mokhammed est son frère ; Fendou est son beau-frère; Abahag et Ouksem sont ses oncles paternels (portant le même nom que le neveu, l'oncle le salue en l'appelant " mon homonyme »). Litni et Aflan sont des voisins appartenant à la tribu noble qui dominait alors le Hoggar. Adhan et Abdelqadir n'ont pas été identifiés

5. Retraduit d'après Galand (Galand, ed. 1999 : 130-134). La traduction adoptée ici, plus proche du mot à mot, est sur un seul point en désaccord avec celle de l'ouvrage : l'expression «il n'y a pas de nouvelles", fréquente dans les lettres, signifie: "nous n'avons pas de nouvelles particulières à vous donner" et non: "nous n'avons pas de nouvelles de vous". 
mais pourraient être d'autres Dag-Ghali. Le nom que ses correspondants donnent à Foucauld est écrit MRBW, où le W représente un $u$ final. C'est le mot français d'origine arabe "marabout ", écrit comme devant se prononcer à la française puisque l'arabe eût exigé un $t$ final.

C'est moi Chikat disant: je salue là-bas mon compagnon le marabout beaucoup beaucoup.

C'est moi Choumekki disant: je salue là-bas le marabout beaucoup je salue ta sour beaucoup beaucoup.

Les gens tous vont bien, il n'y a pas de nouvelles.

C'est moi Ouksem disant je salue là-bas le marabout beaucoup beaucoup.

C'est moi Abahag disant je salue là-bas le marabout beaucoup beaucoup.

C'est moi Litni, le khalifa', disant je salue là-bas le marabout beaucoup beaucoup.

C'est moi Aflan disant je salue là-bas mon vieux le marabout beaucoup beaucoup.

C'est moi Abdelqadir disant je salue là-bas le marabout beaucoup beaucoup.

C'est moi Adhan disant je salue là-bas le marabout beaucoup beaucoup.

C'est moi Adhan disant: je salue là-bas Ouksem beaucoup beaucoup.

C'est moi Ouksem disant: je salue là-bas mon homonyme beaucoup beaucoup.

C'est moi Adhan disant: Mokhammed fils de Chikat a eu un fils. Il n'a pas (encore) reçu de nom.

Sur l'autre face de la feuille, on lit :

C'est le 22 du mois de Sarat

que [la lettre] a été écrite. Les cultivateurs vont bien tous,

les hommes de Tamanrasset.

C'est moi Fendou disant: je salue là-bas le marabout je salue là-bas Ouksem beaucoup beaucoup

Les femmes toutes saluent là-bas le marabout beaucoup beaucoup

Les femmes toutes saluent là-bas Ouksem beaucoup beaucoup.

Cette lettre présente trois traits qu'on retrouve dans à peu près toutes les lettres touarègues : 1) elle se réduit pour l'essentiel à une liste de salutations toutes formulées dans des termes identiques sur lesquels je vais revenir; 2) elle se donne comme émanant de tous ceux dont les destinataires pourraient avoir souci, et s'adresse à tous ceux qui se trouvent à leurs côtés ; 3) elle livre peu d'informations. Détaillons chacun de ces trois traits.

6. Ce mot désigne le représentant à Tamanrasset du chef suprême du Hoggar, lequel réside à l'époque au nord de l'actuel Mali (voir plus loin). 
Tout d'abord les salutations. Leur formulation est la même dans toutes les lettres du Hoggar : c'est moi (litt. : « ceci moi ») A disant je salue là-bas B (awa nek $A$ innân : ehûlegh-în B). La séquence initiale awa nek + nom n'est pas propre aux lettres; elle sert aussi d'incipit aux inscriptions que les forgerons tracent sur les objets qu'ils fabriquent ("Ceci moi Untel l'ayant fait»), ainsi qu'à beaucoup d'inscriptions rupestres dont elle est souvent tout ce qu'on peut en déchiffrer. Le verbe ihal que j'ai traduit par "saluer " est pratiquement toujours présent dans les lettres, au point que le nom dérivé tehult, "salutation", peut avoir le sens de "lettre». Ce n'est pas celui qui désigne les salutations échangées lors d'une rencontre ou d'une visite. Pour celles-ci, on recourt à seslem (causatif d'un verbe eslem emprunté à l'arabe, où l'on retrouve une racine sémitique SLM dont le champ sémantique recouvre les notions de paix, de salut ou de santé). Un homme doit les faire débuter par asalam akhleykem, formule arabe un peu déformée par la prononciation touarègue et qui signifie : le salut soit sur toi. Ce à quoi son interlocuteur lui répond: akhleykem asala $(m)$. Viennent ensuite les formules proprement touarègues (et une femme n'en utilise pas d'autres) : Oyy-iwen? Ma d-tulem? Ma takhlekem? "Vous a-t-il laissés ? (= Dieu vous a-t-il laissé dans la paix ?) ", "Comment allez-vous ? », "Comment vous portez-vous? "À quoi la personne saluée, avant de saluer à son tour, répond à chaque fois: alkher ghas "rien que la paix", en ajoutant parfois des formules d'action de grâces ("Louanges à Dieu!", «Merci à Dieu!»).

Ihal, qui signifierait plutôt "donner le bonjour " ${ }^{7}$, appartient à une catégorie de verbes qui se construisent normalement avec la postposition $d u$ $($ ou $d)$ « ici » ou în « là-bas ». Il est semblable en cela au verbe asu «se déplacer d'un lieu à un autre ", qui signifie "venir " quand il est suivi de $d u$ et " aller » quand il est suivi de în. Avec ihal, la postposition à employer dépend de la position respective de celui qui envoie le bonjour, de celui qui est chargé de le transmettre et de celui qui doit le recevoir. Ainsi, A voyant que $\mathrm{B}$ s'apprête à prendre la route vers un lieu où il rencontrera $\mathrm{C}$, lui dit : " Tu donneras là-bas le bonjour à $\mathrm{C}$ » (tehûlagh-în $C$ ). $\mathrm{B}$, une fois parvenu auprès

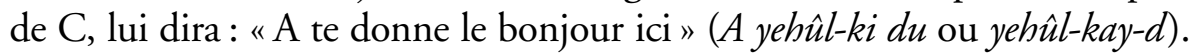
Ordinairement, c'est une fois terminé l'échange de salutations que l'un des partenaires pourra dire à l'autre : "Untel te donne le bonjour ». Autrement dit, on salue (seslem) d'abord son partenaire, puis on lui transmet (ihal) le bonjour d'autrui. Peuvent venir ensuite des informations sur la santé des uns et des autres, et éventuellement des échanges plus libres. Mais, dans une

7. J'ai cependant gardé l'expression "je salue " dans les traductions reproduites ici, pour ne pas trop m'écarter des traductions publiées. 
rencontre sur le chemin entre deux personnes qui ne sont pas très familières, l'échange peut se limiter à son exorde formel: salutation (seslem); transmission du bonjour (tehult); informations sur la santé des proches.

Lorsqu'on transmet ainsi le bonjour, quelqu'un est nécessairement absent. Quand $\mathrm{A}$ donne à $\mathrm{B}$ mission de transmettre son bonjour à $\mathrm{C}$, il est implicite que $\mathrm{C}$ est absent. Quand $\mathrm{B}$ s'acquitte de sa mission auprès de $\mathrm{C}$, A est absent. Foucauld laisse assez bien apparaitre la chose dans le long article que son Dictionnaire consacre à ihal (1951-1952: II, 576-578). Il y fait l'inventaire des personnes auxquelles le verbe peut être conjugué, soit suivi de $d u$ soit suivi de $\hat{\imath}$. La logique implicite selon laquelle s'organise cet inventaire est la suivante: de celui qui parle, de celui à qui l'on parle, ou de celui de qui l'on parle, l'un au moins doit être absent. En particulier, Foucauld remarque que, suivi d'un complément à la première ou à la deuxième personne, ihal ne peut s'employer avec $d u$ "ici " que si le sujet est à la troisième personne. En d'autres termes, lorsque «toi » ou «moi» sommes salués "ici», ce ne peut être que par «lui», absent: de façon exemplaire, la troisième personne est ici la non-personne. À l'inverse, je ne peux dire à quelqu'un que j'ai en face de moi : je te donne le bonjour là-bas. Une telle phrase ne peut être prononcée qu'entre guillemets, lorsque, par exemple, je dis à un voyageur en partance: quand tu verras Untel, tu lui diras de ma part: "Je te donne le bonjour là-bas".

En réalité, il y a bien une situation où je peux m'entendre adresser cette phrase, c'est lorsque je lis une lettre. Toutes les salutations épistolaires que j'ai citées sont de la forme: "Je te donne le bonjour là-bas"; "Je vous donne le bonjour là-bas "; "Ils ou elles te donnent le bonjour là-bas »; ou bien, ce qui est équivalent, "Je donne le bonjour à Untel là-bas », dans une lettre adressée à Untel, ou dont on sait que Untel la lira, ou l'entendra lire. Bien sûr, il est banal de constater que la lettre que je reçois me met en présence d'un absent, mais je trouve remarquable que la forme même des salutations à quoi se réduisent la plupart des lettres fasse apparaitre cette mise en présence de l'absent - comme absent. Et souvenons-nous que ces phrases en principe imprononçables dans une interaction langagière directe, le destinataire d'une lettre ne les lit pas dans un texte, il les entend, ou du moins il entend les séquences sonores dont il les déduit (mais les formules de salutations sont si courantes qu'un lecteur expérimenté les lit en général directement, sans passer par la syllabation intermédiaire). De sorte que le destinataire d'une lettre s'entend dire des phrases qu'on n'entend jamais autrement, soit qu'il les prononce lui-même dans sa lecture à haute voix, soit qu'elles sont prononcées par celui qui déchiffre la lettre pour lui. Comme si tout le contenu de la lettre était entre guillemets. 
Pour en revenir à la lettre adressée à Foucauld, notons que, la graphie y étant assez homogène, on peut penser qu'elle a été rédigée par un scribe unique (ou peut-être deux : un pour chaque face de la feuille) prenant toutes les salutations successives sous la dictée. C'est aujourd'hui encore la situation habituelle. Écrire une lettre est rarement une entreprise solitaire. Le scribe est le plus souvent entouré de plusieurs personnes, dont chacune demande à tour de rôle que ses salutations soient mentionnées et dicte, à l'occasion, telle ou telle nouvelle à ajouter. Quelques indices - ainsi que je l'ai observé moi-même lors de la rédaction de lettres -, me laissent supposer que les épistoliers formaient ici deux cercles. Aux côtés du scribe, les hommes qui envoient nommément leurs salutations, tous nobles ou proches de Chikat. Plus en retrait peut-être, des femmes qui envoient collectivement leurs salutations. Seule, Choumekki, la mère du jeune Ouksem, se nomme. Aucun des cultivateurs - en fait des métayers misérables et, à cette époque, méprisés - n'est nommé, mais il n’en aurait sans doute pas été question s'il ne s'en était pas trouvé au moins un à portée de voix.

Deuxième trait, la lettre est présentée comme émanant de tous ceux dont les destinataires pourraient avoir souci. Ceux qui ne sont pas nommés sont implicitement englobés dans la locution que j’ai rendue par «tous» ou "toutes", traduisant ainsi emdân ou emdânet, formes du verbe emdu (" être fini ", "être inclus jusqu'au dernier ") qui exprime au Hoggar l'idée de totalité. Dans la formule eddûnet emdân, «les gens tous" (mot à mot : "les gens, ils sont inclus jusqu'au dernier»), on pourrait presque dire que emdu est pléonastique. Dérivé de l'arabe duniyya, "le monde, l'univers ", eddûnet signifie: "L'ensemble des gens auxquels nous avons habituellement affaire, qui composent notre monde "; le mot a déjà un sens collectif, que emdu vient encore renforcer. On prend également soin d'adresser la lettre à tous ceux qui pourraient se trouver aux côtés des destinataires lors de sa réception. Ainsi, Choumekki salue la sœur de Foucauld, qu'elle ne connaît pas. Il est vrai qu'elle ne s'adresse pas directement à elle mais dit à Foucauld : je salue ta sœur, et c'est le seul usage de la deuxième personne dans toute la lettre.

On retrouve ce trait dans les lettres du second corpus. Voici une lettre qu'une femme a envoyée aux prisonniers ${ }^{8}$ :

Tihemt fille d'Amoud disant: je salue là-bas Aggour. Les gens [ici] sont vivants tous: Chikat et Chawa, Samana, Tinaqqar, Ghicha, Kounsi et Fadimata.

Je salue là-bas Tassha, et Amastan, Kenan, Moumen.

8. Retraduit d'après Bissuel (1891 : 191-192). Comme pour Galand (Galand, ed. 1999), on a adopté pour ce second corpus une traduction plus proche de l'original touareg. 
Tihemt disant: je salue là-bas Chikat. Dis-lui: les gens [ici] vont bien, ils sont vivants tous. Elle vit Tifist et Kheta et Taqwa et Biben, Kella.

Lépistolière salue tous les destinataires, qu'elle nomme un à un, et envoie à deux d'entre eux, dont le premier est son père, la nouvelle que tous les leurs (la formule est là encore eddûnet emdân) vont bien et sont vivants. Chose assez rare, elle écrit en son seul nom, mais nomme tous ceux dont elle rapporte qu'ils vont bien. Même écrite par un individu, la lettre dit quelque chose de "tous " ceux qui l'entourent.

Dans une lettre écrite par trois des prisonniers, la formule eddûnet emdân est appliquée cette fois aux destinataires. L'épistolier la renforce en disant que sa lettre s'adresse aux vieux comme aux jeunes. Les destinataires ne sont pas nommés, mais présentés comme une collectivité dont le scripteur prend bien soin qu'elle soit couverte de façon exhaustive (Bissuel $1891: 205-206)$ :

C'est Moumen disant: je salue là-bas les gens tous, des vieux jusqu'aux jeunes. Nous allons bien depuis que s'est levée la lune de Sarat.

C'est moi Moumen disant: je salue les jeunes filles toutes. Ne nous oubliez pas. Je salue les violons et leurs maîtresses toutes.

C'est moi Bou Setta disant: je salue là-bas les gens tous, des vieux jusqu'aux jeunes.

C'est moi Bou Setta disant: je salue les jeunes filles toutes. Ne nous oubliez pas. C'est moi Abd es Selam disant: je salue les jeunes filles toutes, les célibataires. Ne nous oubliez pas.

Cette lettre est galante, comme l'est une bonne partie des lettres que les jeunes gens s'échangent volontiers aujourd'hui encore (Aghali-Zakara \& Drouin 1973-1979) : saluer "les violons et leurs maîtresses », et plus encore "les célibataires", c'est avouer combien on souffre d'être privé des réunions nocturnes où garçons et filles avaient alors coutume de s'assembler pour se divertir et deviser.

Troisième trait enfin, la lettre à Foucauld livre peu d'informations. Une ligne dit même qu' "il n'y a pas de nouvelles", c'est-à-dire rien de particulier à annoncer aux destinataires. Puis, tout de même, Adhan, qui a déjà dicté sa salutation, se rappelle que Mokhammed, le frère du jeune voyageur, vient d'avoir un enfant. La naissance n'a pas eu lieu depuis plus de sept jours puisque le nouveau-né n’a pas encore été baptisé. Si Adhan ne s'était pas souvenu de cela, la seule nouvelle apportée par la lettre aurait été que tout le monde à Tamanrasset se porte bien. D'autres lettres en apportent encore moins. Ainsi ces quatre lignes que Chikat et ses frères ont écrites à la même époque (Galand, ed. 1999 : 150-152) : 
C'est moi Chikat disant: je salue là-bas le chef, le marabout.

C'est moi Ouksem disant: je salue là-bas le marabout beaucoup beaucoup.

C'est moi Abahag disant: je salue là-bas le marabout beaucoup beaucoup

Tu nous manques.

Le "Tu nous manques" venant après trois formules de salutations n'apportait pas vraiment de "nouvelles" au destinataire, mais lui disait simplement l'affection de ses correspondants. Il est vrai que ces quatre lignes n'allaient peut-être pas seules car elles apparaissent au recto d'une enveloppe adressée à Foucauld chez sa sœur. On peut penser que celui-ci avait laissé des enveloppes toutes prêtes à ses amis touaregs avant de prendre la route. Ses correspondants auraient ajouté ces mots sur l'enveloppe après l'avoir cachetée. Les éditeurs n'excluent pas que la lettre contenue dans l'enveloppe ait été tout simplement celle que j'ai citée plus haut. Si c'était le cas, lettre et enveloppe n'auraient contenu au bout du compte que bien peu d'informations.

Certaines lettres apportent des informations moins attendues, comme la mort ou la maladie d'un proche, mais cette annonce ne modifie pas leur tonalité générale. Elle apparaît dans le chapitre des nouvelles, après des salutations qui restent identiques à ce qu'elles sont d'habitude.

On a aussi quelques lettres très brèves, où la seule information est une demande, ou un remerciement (Galand, ed. 1999: 186, 190, 199) :

Tedawit disant: je salue là-bas le marabout beaucoup beaucoup je salue là-bas ta sour beaucoup, merci à vous, merci au marabout et à sa sour de ne pas nous avoir oubliés. Les remèdes sont arrivés, je vais guérir maintenant. C'est le papier qui fait défaut. Tedawit disant: je salue là-bas Ba-Khammou beaucoup.

C'est moi Mokhammed fils d'Akroud disant: je salue le marabout. Dis-lui ${ }^{9}$ : donne une pièce d'étoffe à Eqqesen.

C'est moi Ouksem, je salue le marabout beaucoup beaucoup. Je veux deux pioches. Donne-les à Aggag.

Je ne pense pas que Foucauld était en France au moment où ces lettres lui ont été envoyées. C'est exclu pour la première puisque Ba-Khammou, le deuxième destinataire de la lettre, n'a jamais quitté le Sahara. L'épistolière salue la sœur de Foucauld, mais il s'agit vraisemblablement de salutations qu'elle demande à celui-ci de retransmettre dans sa propre correspondance. Relevons au passage les remerciements qu'elle adresse à ses correspondants, et dont une traduction littérale serait: "Merci au marabout et à sa sœur du non-oubli» (iba n tettawt). Quant aux deux

9. Cet impératif s'adresse à la lettre elle-même. 
autres lettres, elles ont pour objet de menues sollicitations dont on voit mal qu'un homme en voyage à l'étranger ait pu en être l'objet. J'imagine que Mokhammed et Ouksem, en même temps qu'ils chargeaient Eqqesen et Aggag de transmettre oralement leurs salutations et leurs demandes, leur ont confié ces petits billets qui venaient à l'appui du message oral. L'écrit est ici inséparable d'une communication orale, d'abord parce qu'il accompagne et appuie un message oral, ensuite parce que ceux qui écrivent ont vu il y a peu de temps et verront sous peu celui à qui ils écrivent.

Toutes ces lettres impersonnelles posent des problèmes d'authentification. On peut certes dire qu'elles sont signées puisque les épistoliers s'y nomment, mais cette mention de son nom par le destinateur n'est pas en mesure de remplir l'une des fonctions dévolues à la signature dans les sociétés faisant un usage courant de l'écriture : authentifier l'écrit où elle figure. Lorsque l'épistolier veut donner à son correspondant la certitude qu'il est bien l'auteur de la lettre, il adjoint à son écrit des indices connus d'eux seuls, et que les Touaregs appellent tametert. Ainsi dans une des lettres du second corpus, envoyée à des compagnons restés en captivité par un prisonnier fraîchement libéré, on trouve ces mots (Bissuel 1891 : 200) : "Pour que vous sachiez l'indice : la boule de chiffons". L'éditeur explique qu'il s'agit d'une balle dont les prisonniers jouaient entre eux sur l'une des terrasses du fort où ils étaient incarcérés. Dans une autre lettre du même corpus, adressée à un nommé Kenan avec qui le scripteur s'était rendu en France, on lit: "Afin que tu saches l'indice, Kenan, lorsque nous sommes allés à Marseille, le soir, un homme nous a dit : “Aucun Touareg n’est venu ici avant vous" "(Ibid.: 208).

\section{Lettres contemporaines}

Ces traits se retrouvent, sous une forme un peu différente, dans les lettres qu'on m'a montrées au Niger. La formule d'ouverture y devient: "C'est moi A disant : je fais [mes] salutations [à] B, C, D beaucoup beaucoup et [aux] gens tous" (awa nek A yennân: tibulawén ikânnin B C D wullen wullen entén aghalak kullu - où tihulawén est le pluriel du tehult déjà mentionné). En général, le scripteur enchaîne sur les formules de salutation normalement utilisées dans le face-à-face (Oyy-iwen? Mad-tulem ?, etc.). Autrement dit, après l'envoi du bonjour réservé aux absents, il salue ses correspondants comme s'ils étaient présents. Peuvent venir ensuite des informations sur la santé des uns et des autres semblables à ce qu'on trouve dans les lettres du Hoggar ou dans la conversation. Les lettres nigériennes reprennent donc les formules de l'échange en face-àface, mais en introduisant dans l'ordre de leur succession une modification 
qui me paraît pouvoir s'interpréter ainsi : ceux qui se font face ne se transmettent le bonjour des absents qu'après avoir échangé les salutations qu'ils se doivent l'un à l'autre, tandis qu'un épistolier ne peut en venir à de telles salutations qu'une fois que le bonjour envoyé à ses correspondants les a fictivement mis en sa présence.

La locution entén aghalak kullu, "et [aux] gens tous ", répond au verbe emdu des lettres du Hoggar. On en retrouve l'équivalent dans les lettres en français, dont il faut maintenant parler. Les jeunes gens scolarisés, qui souvent se sentent plus habiles à manier l'alphabet latin que les tifinagh, écrivent volontiers leurs lettres en français, comptant que leurs destinataires parviendront toujours à trouver un interprète. Il m'est arrivé, lorsque j'étais accueilli dans un campement, qu'on me prie de lire de telles lettres, reçues parfois depuis plusieurs semaines et attendant encore d'être déchiffrées. À l'inverse, mes amis me demandaient parfois d'écrire en français à des parents scolarisés dont ils pensaient qu'ils se débrouilleraient mieux avec le français qu'avec les tifinagh. J'ai moi-même reçu de telles lettres. Voici l'exorde de deux d'entre elles, tout à fait semblables à celles que les Touaregs s'écrivent entre eux lorsqu'ils utilisent le français. L'auteur, auquel je donnerai ici le nom de Mokhammed, avait suivi sa scolarité jusqu'au certificat d'études et était employé des postes à Agadez.

Cher ami D. jai t'envoi celle-ci pour te mettre au courant de mes nouvelles toute en espérant les tiennes en échange, nous somme en parfaite santé ainsi la famille. La famille Achidou vous salue très bien elles vont très bien pour l'instant aucun mal. Tout ms salutations à ta famille sans oubliers personne.

cher ami Dominique j'ai tenvoie celle-ci pour te mettre au courant de me nouvelles tout en spérant les tiennes en échange. J'ai suis en parfaite santé et moi la famille, nous souhaitons une bonne heureuse année 1983 toit et ta famille les sours le père la mère sans oublier personne.

Le « sans oublier personne» dont Mokhammed fait suivre ici l'énumération des proches de son correspondant transpose assez fidèlement le entén aghalak kullu qui, dans les lettres en touareg, suit l'énumération des destinataires. Mais le français dit plus ouvertement un souci que le touareg laisse implicite: il faut que personne, parmi les destinataires et leurs proches, ne se sente oublié. Et c'est bien ainsi que mes amis mont commenté aussi bien la formule touarègue que sa transposition française. Se voir oublié par une lettre est un chagrin qu'un bon épistolier se garde d'infliger, surtout qu'il sait bien que la victime l'éprouverait sous le regard de tous. Rappelons-nous, en effet, que ni l'écriture ni la lecture d'une lettre ne 
sont des actes solitaires. Le scribe, qui doit déjà veiller à associer à sa lettre tous ceux qui l'entourent et transcrire scrupuleusement leurs salutations, doit aussi faire en sorte qu'à la réception de la lettre aucun des présents ne puisse se sentir exclu du cercle des auditeurs. Au fond, les lettres touarègues ne disent rien d'autre que: "Nous qui faisons cercle autour $\mathrm{du}$ scribe, nous ne vous oublions pas, vous qui faites cercle autour du lecteur»; message dont le corollaire parfois explicité est : "Et vous, ne nous oubliez pas». Telle est finalement la principale "nouvelle» qu'elles sont destinées à transmettre, et les informations dont elles sont pour le reste si avares comptent moins que cette nouvelle-là. À la différence des livres qu'ils voient écrits en arabe ou en français, les Touaregs n'attendent pas de leurs lettres qu'elles disent quelque chose sur le monde, mais qu'elles conjurent la menace de l'oubli - ou, pour le dire dans leur langue, qu'elles garantissent le non-oubli (iba $n$ tettawt) des absents.

Et si les informations sont minces, la crainte de l'oubli se dit avec insistance. J'ai noté plus haut combien le eddûnet emdân du Hoggar était pléonastique. Le entén aghalak kullu du Niger ou son équivalent français "sans oublier personne" le sont plus encore puisque ces formules concluent des énumérations où le scripteur a déjà pris soin de n'omettre aucun nom. Sans doute, précautions prises contre la malencontre d'un oubli toujours possible, ces formules visent-elles à inclure dans l'énumération ceux-là mêmes dont le nom aurait par inadvertance été oublié, mais il me semble qu'elles traduisent encore un autre souci, plus diffus. Car cette propension au pléonasme, l'écrivain irlandais John Synge l'avait déjà rencontrée dans les îles Aran à la fin des années 1880. Il lisait une lettre reçue de Michaël, un jeune homme connu lors d'un séjour précédent et qui s'était depuis installé sur le continent. Nous ne sommes pas si loin des Touaregs qu'on pourrait le croire. Michaël, tout comme mon ami Mokhammed, a suivi quelques années de scolarité et est allé gagner sa vie loin des siens. Il écrit dans un gaélique solennel et guindé, différent du français de Mokhammed, mais très semblable au français un peu grandiloquent des Nigériens qui ont poussé leurs études jusqu'au lycée. Voici ce qu'il écrit (Synge 1996 [1921] : 91) : "Comment vont ma mère et mes trois frères et mes sœurs, et n'oubliez pas le blanc Michaël [un homonyme] ni le pauvre petit enfant ni la vieille femme grise ni Rory. J'en viens à oublier tous mes amis et tous mes parents». Et Synge de trouver «singulier qu'il s'accuse ainsi d'oubli après avoir demandé nommément des nouvelles de toute la famille ». S'inquiéter de n'avoir oublié personne après avoir salué nommément tous ceux qu'il ne fallait pas oublier est tout aussi singulier. Synge a ce commentaire : "Je suppose que la nostalgie du foyer qu'il avait au début s'atténue peu à peu et qu'il considère son indépendance et son bien-être 
comme une trahison envers les siens ". C'est assurément une interprétation possible, mais la singulière attitude de Michaël, tout comme les formules touarègues tiennent peut-être aussi à la situation épistolaire elle-même. L'effort de mémoire si léger soit-il que je dois faire au moment d'aligner sur le papier les noms de mes absents me rend conscient de ce qu'ils ne sont pas constamment présents à mon esprit. Me souvenir d'eux me fait sournoisement sentir que l'oubli déjà les menace, car je vois bien qu'à l'instant d'avant je ne pensais pas spécialement à eux, préoccupé d'affaires où ils ne sont pas. Bien sûr, je me souviens encore d'eux puisque je viens d'écrire leurs noms mais leur image en moi, qui fluctue et pâlit parfois, n'a pas la ferme présence du nom tracé sur ma feuille. En voyant sur le papier la preuve écrite qu'il n'avait pas oublié les siens, Michaël gémissait de ce que la netteté même de cette preuve la faisait se retourner contre elle-même. Cette inexorable érosion de la mémoire que l'écriture même des lettres rend sensible, les épistoliers touaregs la conjurent par des formules qui ne veulent rien dire d'autre que : "Je crois n'oublier personne mais d'avoir de mon calame tracé la liste de vos noms m'en fait douter, alors pardonnez-moi si le sournois oubli déjà m’aveugle ».

Plus encore qu’à celle de Michaël, les lettres de Mokhammed font penser aux lettres d'émigrés béarnais étudiées par Anne Bruneton-Governatori et Bernard Moreux (1997), ou à celles que ses amis tziganes dictaient à Patrick Williams (1997). Le "j[e] t'envoi[e] celle-ci pour te mettre au courant de me[s] nouvelles tout en espérant les tiennes en échange " par lequel il commence ses lettres a certainement été repris de modèles français mis en circulation à l'époque de la colonisation et adoptés depuis par les Nigériens scolarisés. En revanche, on a vu que "sans oublier personne " transpose une formule touarègue. De même, "La famille [d']Achidou vous salue très bien elles vont très bien pour l'instant aucun mal » traduit presque mot pour mot la transmission du bonjour et l'envoi de nouvelles rencontrés dans les lettres précédentes. Mokhammed mêle donc un modèle épistolaire autochtone à un modèle venu de France, mais les deux modèles se ressemblent de toute façon: mêmes salutations formulaires, mêmes références stéréotypées à la santé des proches. On associerait volontiers les Touaregs à l'internationale épistolaire que Bruneton-Governatori et Moreux mettaient en évidence à partir de corpus européens du XIX et du XVIII siècle, ainsi que de quelques lettres antiques ou médiévales.

Le caractérisant comme "populaire", ces auteurs opposent le modèle qu'ils ont dégagé à celui dont les manuels de correspondance ont peu à peu diffusé l'idéal à partir du XVIII siècle. Les deux modèles se veulent l'écho de l'interaction langagière, mais le second suppose en réalité une pleine maîtrise de l'écrit et, loin de mimer l'échange verbal commun, 


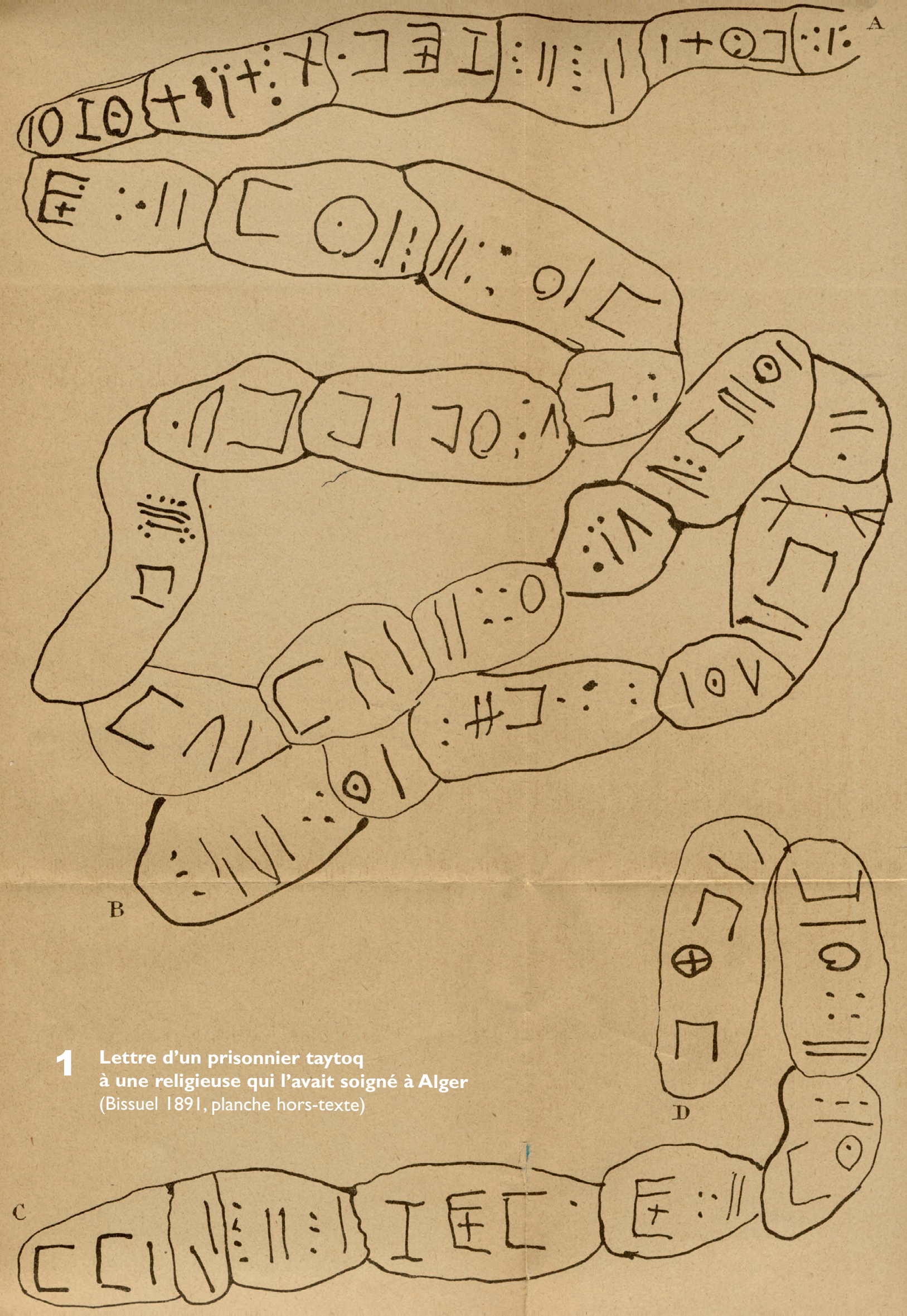




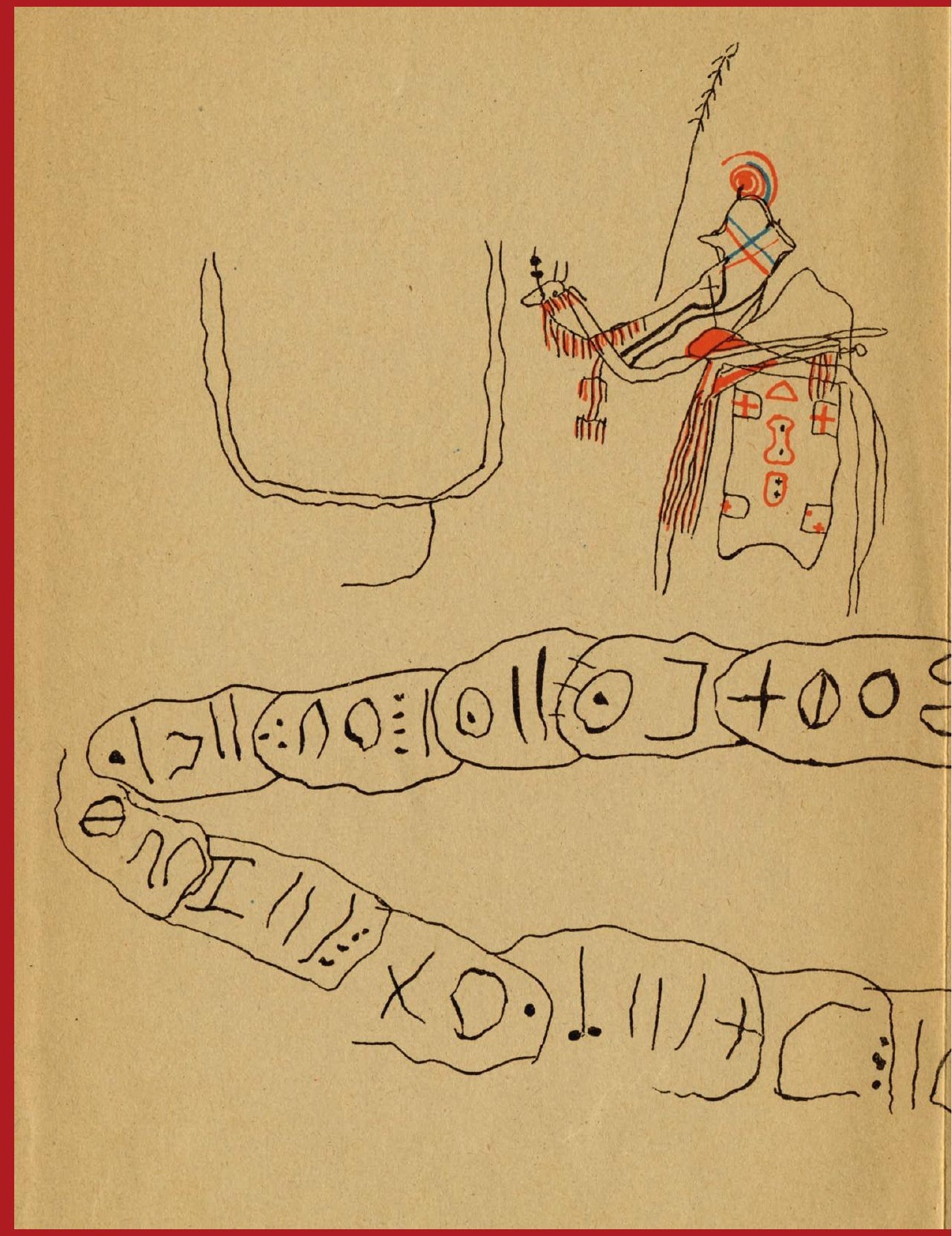




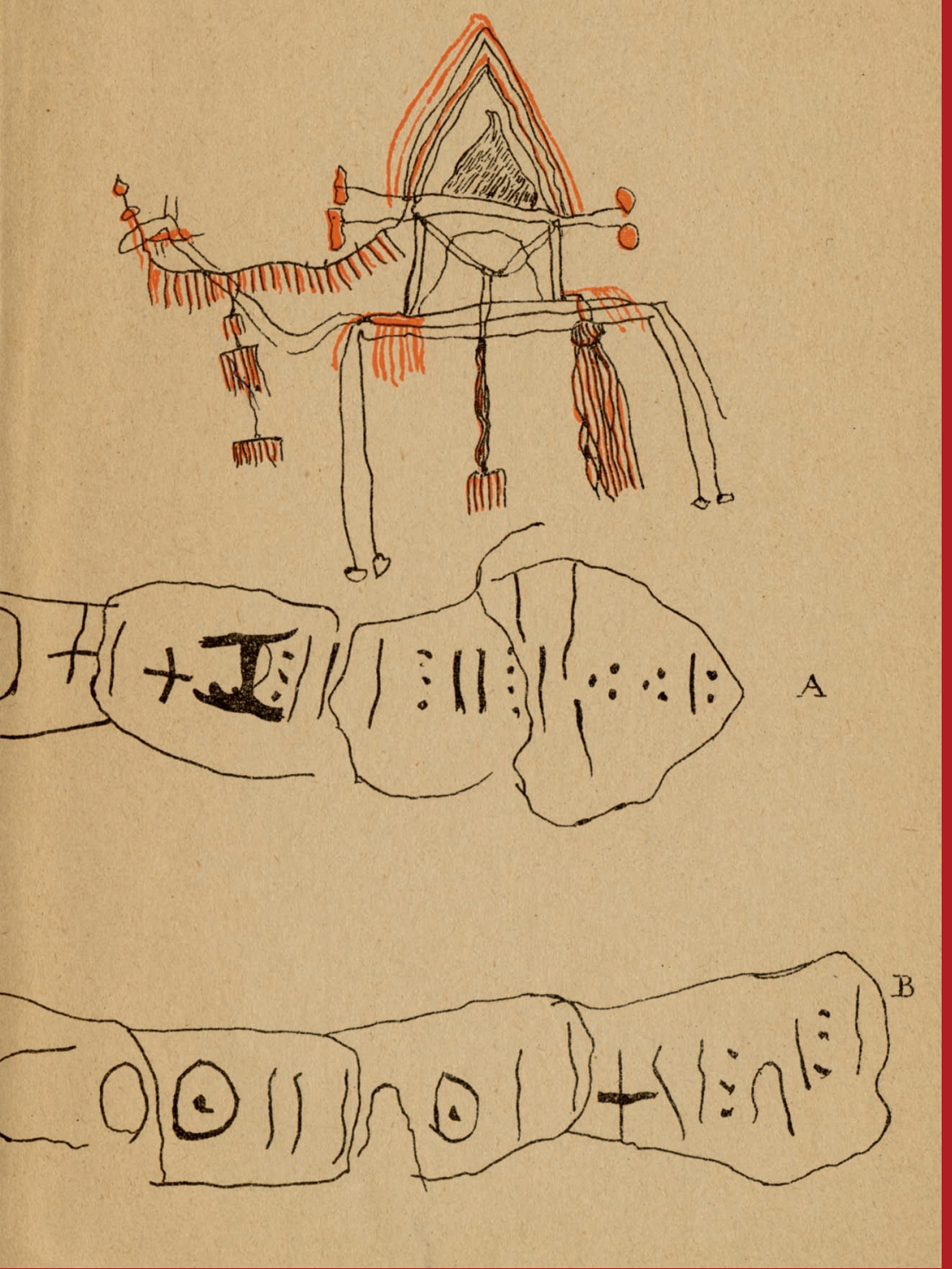

2

Lettre

d'un prisonnier taytoq à un officier français

(Bissuel 1891, planche hors-texte) 


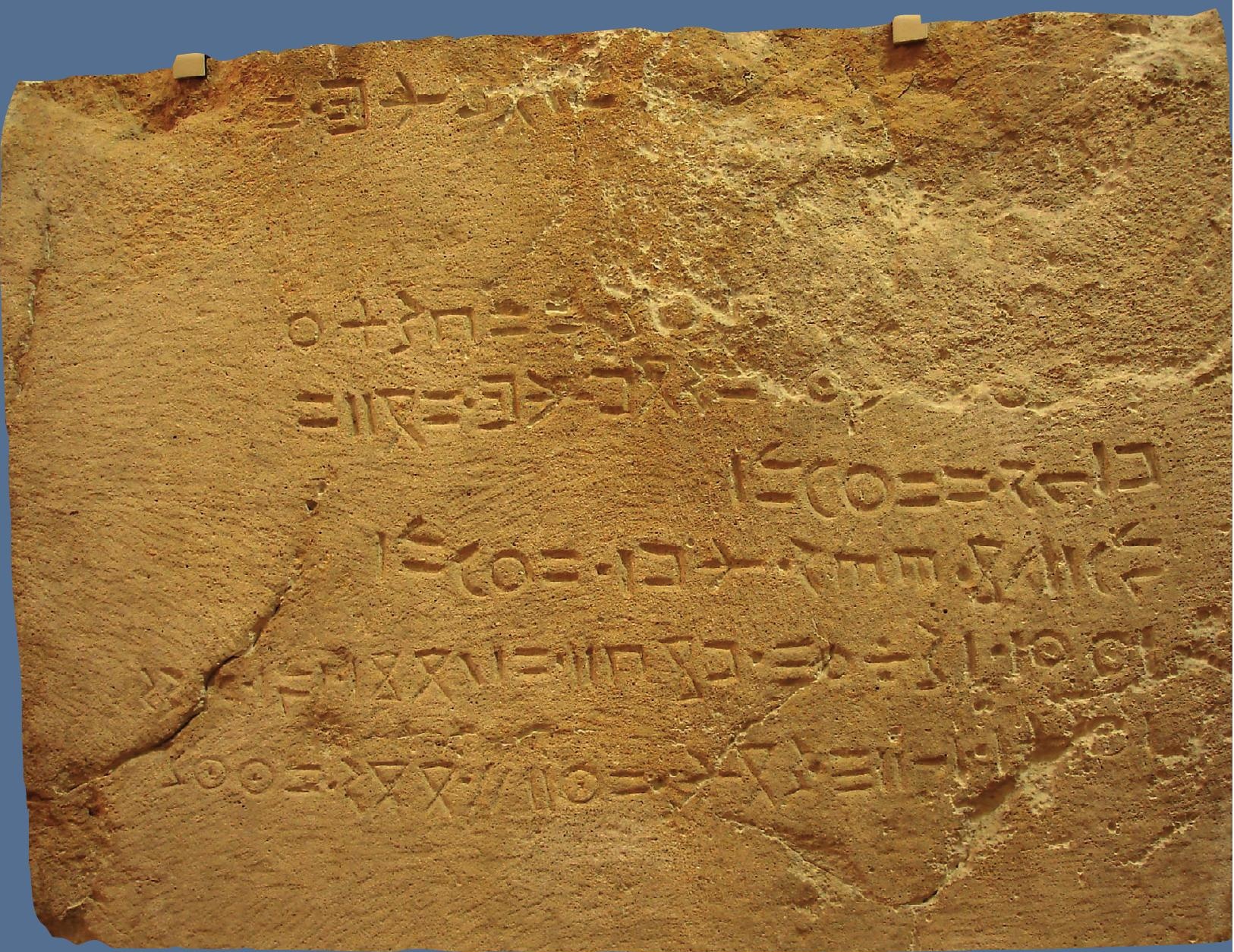

3

Inscription libyque dite du mausolée d'Atban,

Dougga, ca lle siècle av. J.-C.

(Cl. Sophie de Beaune) 
transpose dans la pratique épistolaire l'art de la conversation qui se répandait alors dans les milieux lettrés (Fabre 1997: 21). De façon comparable, les petits messages commentés jusqu'ici different de lettres plus recherchées dont je n'ai encore rien dit. Elles sont fort rares et l'une d'entre elles suffira à éclairer mon propos. Elle a été écrite en janvier 1914 par Moussa agg Amastan, le chef suprême (amenokal) des Touaregs du Hoggar, qui nomadisait alors au nord de l'actuel Mali. Foucauld revenu de France s'est arrêté à Tamanrasset, tandis que le jeune Ouksem a continué sa route jusqu'aux tentes de l'amenokal (Galand, ed. 1999: 161-163) :

C'est moi Moussa, amenokal de l'Ahaggar,

Disant: je salue là-bas mon ami affectionné, mon compagnon le marabout, serviteur de Jésus, beaucoup beaucoup. Louanges à Dieu pour ta santé. Tu nous manques fort.

J'ai vu ton jeune Ouksem, il nous a donné des nouvelles, merci beaucoup. Je t'ai écrit une lettre que j'ai fait porter au lieutenant à Boughessa, il te la transmettra.

Nous, nous allons bien si tu vas bien. Elle te salue ici Tedawit, ainsi que Akhamouk, Litni, et tous, et Rakhma.

Je salue là-bas le général Laperrine, Nieger, et la France toute.

Le lieutenant avait l'intention de me rejoindre auprès de mes tentes et nous serions allés ensemble dans l'Ä̈r [au nord du Niger]. Maintenant il a changé d'avis, il m'a donné un délai de deux mois pour que je le rejoigne à $T i-n-Z a w a t e n$ avec tous mes gens. Il reste quarante jours avant la date fixée.

J'écris cette lettre le 7 du mois de Settafet.

Ne me délaisse pas. Je te demande une chose : prie beaucoup pour moi. Salut (asalam).

Le papier manque [et je ne peux donc pas t'écrire davantage].

Moussa n'omet rien des salutations usuelles, mais il y met un ton qui n'appartient qu'à lui. Il appelle Foucauld successivement "mon émeri » et " mon ämîdi», termes qui dérivent respectivement de är "aimer" et de iddew "aller de compagnie avec". Le Dictionnaire de Foucauld (19511952: IV, 1549) les rend respectivement par "ami affectionné" et "compagnon ", précisant même à propos du premier : "signifie non les simples liaisons ni les amitiés ordinaires, lesquelles s'expriment par ämîdi "compagnon", mais un amour profond, com[me] celui qui existe entre 2 amis intimes ou entre un h. et une f. qui s'aiment passionnément". Chikat et son fils Ouksem, à qui Foucauld portait une affection payée de retour, utilisent indifféremment les deux termes; Moussa, en utilisant successivement l'un puis l'autre, apparaît soucieux de charger émeri de tout 
son sens. Il est aussi le seul à donner à Foucauld le beau nom de «serviteur de Jésus" (akli n-Ghissa), attention à laquelle il sait certainement que son destinataire est sensible. Dans une autre lettre, il l'appelle Shaghl "Charles ", transcrivant le $r$ de Charles par la vélaire constrictive $g h$ et non l'apicale vibrante $r$. Pour une oreille française, ces deux phonèmes correspondent à peu près à un $r$ grasseyé et à un $r$ roulé. En général, les Touaregs entendent le $r$ français comme une apicale et non comme une vélaire, alors que les Français s'essayant à la langue touarègue auraient plutôt tendance à le transcrire par une vélaire. C'est dire que Moussa écrit le prénom de Foucauld comme celui-ci l'entendait et non pas comme, selon toute probabilité, il l'entendait lui-même. Marque tout à la fois d'affection et de courtoisie.

Le "Louanges à Dieu pour ta santé » qui suit les formules habituelles de salutation équivaut, en plus chaleureux, au «[Dieu] vous a-t-il laissé [dans la paix] ?» que les épistoliers nigériens placent dans leurs lettres à cet endroit. Moussa est le seul à lier la mention de sa santé à celle de son destinataire: "Nous allons bien si tu vas bien » rappelle, en plus affectueux, le «Je vais bien et j'espère que la présente vous trouvera de même » si fréquent dans les lettres européennes. "Je salue là-bas le général Laperrine, Nieger, et la France toute ", par lequel le chef touareg salue les officiers français qu'il a eu l'occasion de connaître, est une variante altière du "Je salue là-bas les gens tous" dont se contentent ses vassaux dag-ghali. Par ailleurs, ce qu'il dit de ses démêlés avec «le lieutenant» (le chef du peloton méhariste du Hoggar) n'a pas le caractère un peu attendu des nouvelles apportées par les autres lettres du corpus. Enfin, le asalam (normalement réservé aux salutations en face-à-face) dont sa lettre offre l'unique occurrence est précédé d'une phrase où se marque, avec un abandon dont les Touaregs offrent rarement l'exemple, tout ce que le vieil ermite représente pour le pieux amenokal ${ }^{10}$.

D'autres personnes envoient leur bonjour à Foucauld, mais Moussa ne leur donne pas vraiment la parole. Les lettres précédentes commençaient toutes par une série de "Moi, Untel, je te salue là-bas ». Chacun envoyait «là-bas" (în) en son nom des salutations que le scribe se contentait de transcrire. Dans cette lettre, le scribe (dont je ne sais s'il est Moussa lui-même ou un secrétaire écrivant sous sa dictée) a écrit: "Tedawit, Akhamouk, etc. te saluent ici. "C'est-à-dire : ils me confient "ici» (d) le bonjour qu'ils me chargent de te transmettre là-bas. Se posant comme l'unique interlocuteur de Foucauld, il tient à rester en tête-à-tête avec lui, imposant à ceux qui ont quelque chose à dire à son correspondant de passer par son intermédiaire. À moins qu'il ne se représente comme lui

10. Sur les relations entre les deux hommes, cf. Casajus (2009), dont j’ai repris quelques éléments ici. 
faisant face, car il lui parle comme si, ayant fait le voyage jusqu’à lui, il lui transmettait « ici » le bonjour de son entourage. Subvertissant les formules traditionnelles de salutation, le chef touareg a su faire passer la force de ses sentiments dans le rigide carcan des conventions épistolaires.

Est-ce à dire que, avec son versant populaire et son versant aristocratique, le paysage épistolaire est analogue en pays touareg à ce qu'il est devenu en Europe après le XVIII ${ }^{\mathrm{e}}$ siècle ? Il existe de fait quelques analogies. Tout comme les épistoliers béarnais considérés par Anne Bruneton-Governatori et Bernard Moreux (1997), Mokhammed n'a acquis de la culture française que quelques éléments, comme eux il a reçu un enseignement élémentaire dans une langue qui n'est pas sa langue maternelle, et comme beaucoup d'entre eux sans doute, un modeste emploi en ville l'a éloigné de ses origines rurales. En revanche, pour les Touaregs qui usent de leur vieil alphabet, l'analogie ne tient plus. Au contraire de ce qu'il en est pour nos épistoliers populaires, l'écriture n'a pas à leurs yeux une dignité à laquelle il leur faudrait péniblement se hisser. Aucun stigmate ne s'attache à son ignorance, et sa maîtrise n'introduit pas à un savoir refusé à l'ignorant, puisqu'il n'y a pas de livres écrits en tifinagh. Ceux qui manient l'alphabet touareg avec le plus d'aisance n'écrivent pas davantage pour autant; tout au plus aiment-ils faire étalage de leur virtuosité dans des jeux de société où l'on demande à son partenaire de déchiffrer des inscriptions tracées sur le sable. De plus, avec ses lettres géométriques, son consonantisme, l'écriture touarègue se prête mal à la transcription fluide de la parole. Les jeunes créateurs d'alphabets ont précisément voulu lui donner la fluidité qu'ils ont expérimentée dans les écritures apprises au lycée ou à la medersa, mais les instruments qu'ils ont ainsi forgés n'ont plus grand-chose de commun avec celui que leurs contribules non scolarisés manient depuis toujours. Ceux-ci appliquent au calame et au papier une vieille écriture conçue pour le burin et la pierre, et les plus virtuoses d'entre eux y sont encore maladroits. Cette maladresse ne fait cependant pas d'eux des demi-lettrés, étant consubstantielle à l'écriture elle-même. Et tout malcommode qu'on puisse trouver l'instrument qu'ils manient avec tant de peine, il remplit parfaitement la seule fonction qu'ils lui assignent : préserver ce que l'oubli menace. Quant à la roide formularité de leurs épîtres, nous avons vu qu'elle transpose la formularité des salutations en face-à-face ; elle tient autant aux usages oraux qu’à la maladresse des scripteurs.

Fort bien, me dira-t-on, mais l'exemple de Moussa montre pourtant que l'usage des tifinagh ne condamne pas automatiquement à la maladresse. À cela je répondrais que l'aisance de Moussa lui vient peut-être de sa connaissance d'écritures et de traditions épistolaires étrangères. Ses fonctions lui imposent d'entretenir une abondante correspondance 
en arabe avec les officiers français et avec ses vassaux. Il dispose pour cela d'un secrétaire car, bien que comprenant l'arabe, il ne l'écrit pas. La lettre citée ici, dont l'onctuosité rappelle celle des épistoliers arabophones du Sahara d'alors, s'inspire certainement du modèle qu'il suit dans cette correspondance. Une lettre en arabe qu'il a envoyée à Foucauld le 20 septembre 1910 nous est d'ailleurs parvenue. Élégante et affectueuse, elle est assez comparable à celle que j'ai examinée ici. De son côté, Foucauld a l'habitude de lui écrire, son diaire en témoigne. Ces lettres sont perdues, mais le brouillon d'un message où l'ermite a traduit en touareg à son intention les instructions d'un officier pourrait en donner quelque idée. Retrouvé dans les papiers de Foucauld après sa mort, ce texte ne ressemble guère aux lettres commentées ici. Il est riche en informations et pauvre en salutations, au point d'apparaître comme un peu impoli selon les normes locales : en un mot, il est écrit à la française (Galand, ed. 1999: 209). La cursive arabe que Moussa a vu manier sous sa dictée, les longues lettres touarègues qu'il a reçues de Foucauld, tout cela lui a appris que l'écriture pouvait servir à d'autres tâches que celles que lui réservent traditionnellement les Touaregs. Comme l'ont fait après lui les militants berbérophones, il a transposé dans son usage des tifinagh une expérience venue de la fréquentation d'autres écritures, sans attenter toutefois au vieil instrument reçu de ses pères.

Tous ceux qui se sont intéressés aux écritures ordinaires savent combien elles se situent aux frontières entre l'écrit et l'oral, dans une zone où l'écrit agit au sein d'un monde de parole et inversement. Sous ce rapport, les Touaregs nous sont apparus comme exemplairement frontaliers, eux qui utilisent leur alphabet en hommes de la parole autant qu'en hommes de l'écrit. Frontaliers, ils le sont aussi au sens qu'un Gelb aurait peut-être placé leur écriture sinon parmi les syllabaires, du moins sur la limite qui les sépare des alphabets proprement dits. Ils le sont encore au sens que cette écriture autochtone cohabite chez eux avec deux écritures allochtones, cohabitation qui est aussi celle de plusieurs rapports à l'écrit. Ils le sont enfin en un autre sens. Nomades que leurs transhumances ou leurs voyages séparent souvent de leurs proches, ce qu'ils attendent de l'écriture est avant tout de les rendre présents à leurs absents : la majeure partie de ce qu'un Touareg écrit $i c i$ sera lu là-bas. Et encore ces écrits destinés à franchir les invisibles frontières de la distance et du temps ne parviennent-ils pas directement à leurs destinataires puisque ceux-ci ne les reçoivent le plus souvent que par l'intermédiaire d'un lecteur qui les déchiffre pour eux et que, même s'il est seul, le destinataire doit séparer en lui le déchiffreur et le lecteur. Tout cela marque entre 
leur vieil alphabet et les autres alphabets qu'ils connaissent une frontière qu'un Moussa agg Amastan a cependant franchie jadis et que les militants berbérophones brûlent aujourd'hui d'abolir. S'ils y parviennent un jour, les Touaregs verront se modifier non seulement leur rapport aux tifinagh mais aussi leur rapport à l'écriture elle-même.

Centre national de la recherche scientifique Centre d'études des mondes africains, Ivry-sur-Seine casajus@ivry.cnrs.fr

MOTS CLÉSIKEWORDS: alphabet - écriture libyco-berbère/libyco-berber script - art épistolaire/letter-writing - syllabaire/sillabary - écriture consonantique/vowless alphabet Touaregs/Tuaregs. 
Aghali-Zakara, Mohamed

1994 "Graphies berbères et dilemme de diffusion : interaction des alphabets latins, ajami et tifinagh", Etudes et documents berbères $11: 107-121$.

Aghali-Zakara, Mohamed \& Jeanine Drouin 1973-1979 "Recherches sur les tifinagh", Groupe linguistique d'étude du chamitosémitique 18-23 (2) : 245-272 et 279-292.

1979 Traditions touarègues nigériennes. Paris, L'Harmattan.

2007 Inscriptions rupestres libyco-berbères. Genève, Droz.

\section{Albert, Jean-Pierre}

1993 "Écritures domestiques ", in Daniel Fabre, ed., Écritures ordinaires... : 37-94.

\section{Alvarez-Pereyre, Frank}

1989 «Lois et paroles millénaires: la Thora et la Michna entre texte écrit et oralité ", in Graines de parole: puissance du verbe et traditions orales. Textes offerts à Geneviève Calame-Griaule. Paris, CNRS : 23-38.

\section{Bissuel, Henri}

1891 Le Sahara français. Alger, Adolphe Jourdan.

\section{Bruneton-Governatori, Anne \& Bernard Moreux}

1997 «Un modèle d'écriture populaire : les lettres d'émigrés béarnais ", in Daniel Fabre, ed., Par écrit... : 79-103.

\section{Camps, Gabriel}

1973 "Recherches sur les plus anciennes inscriptions libyques de l'Afrique du Nord et du Sahara ", Bulletin archéologique $d u$ CTHS nouv. ser. 10-11 : 143-166.
Casajus, Dominique

2000a «L'errance d'Imrû'l-Qays :

poésie arabe et poésie touarègue ", Journal des Africanistes 72 (2) : 139-151.

2000b Gens de parole. Langage,

poésie et politique en pays touareg.

Paris, La Découverte.

2009 Charles de Foucauld. Moine et savant.

Paris, CNRS Éd.

2011 «Déchiffrages: quelques réflexions sur l'écriture libyco-berbère ", Afriques.

Débats, méthodes et terrains d'histoire

[http ://afriques.revues.org/688].

\section{Chaker, Salem \& Slimane Hachi}

2000 «À propos de l'origine et de l'âge de l'écriture libyco-berbère : réflexions du linguiste et du préhistorien ", in Salem Chaker \& Adam Zaborski, Études berbères et chamito-sémitiques. Mélanges offerts à Karl-G. Prasse. Louvain, Peeters : 95-111.

\section{Coninck, P. de \& Lionel Galand}

1960 «Un essai des Kel-Antessar pour améliorer l'écriture touarègue ", Groupe linguistique d'étude du chamito-sémitique 8 : 78-83.

Fabre, Daniel

1997 «Seize terrains d'écriture », in Daniel

Fabre, ed., Par écrit. . . : 1-56.

Fabre, Daniel, ed.

1993 Écritures ordinaires. Paris, Centre national d'art et de culture GeorgesPompidou, BPI.

1997 Par écrit. Ethnologie des écritures quotidiennes. Paris, Éd. de la Maison des sciences de l'homme.

Février, James Germain

1959 Histoire de l'écriture. Paris, Payot. 
Foucauld, Charles de

1951-1952 Dictionnaire touareg-français.

Dialecte de l'Ahaggar. Paris, Imprimerie nationale. 4 tomes.

Galand, Lionel

1976 "La notion d'écriture dans les parlers berbères ", Almogaren 5-6 : 3-97.

2001 «Un vieux débat: l'origine

de l'écriture libyco-berbère", La lettre

du Répertoire des inscriptions

Libyco-Berbères $7: 1-3$.

Galand, Lionel, ed.

1999 Lettres au marabout. Messages touaregs au Père de Foucauld. Paris, Belin.

Gelb, Ignace J.

1973 Pour une théorie de l'écriture.

Paris, Flammarion.
Lahire, Bernard

2001 L'Homme pluriel. Les ressorts

de l'action, Paris, Hachette Littératures.

Pichler, Werner

2007 Origin and Development

of the Libyco-Berber Script.

Köln, Rüdiger Köppe.

Synge, John Millington

1996 [1921] Les Îles Aran.

Trad. par P. Leyris. Paris, Payot.

\section{Williams, Patrick}

1997 «L'écriture entre l'oral et l'écrit:

six scènes de la vie tzigane en France", in Daniel Fabre, ed., Par écrit... : 59-78.

RÉSUMÉ/ABSTRACT

Dominique Casajus, Écritures ordinaires en pays touareg. - Les Touaregs disposent d'un vieil alphabet dont les caractères reçoivent le nom de tafineq, terme qui pourrait être un souvenir du mot dont les Grecs désignaient les Phéniciens. Cet alphabet autochtone, qui dérive d'alphabets - dits libyques - dont la plus ancienne attestation datée remonte au milieu du IIe siècle avant J.-C., cohabite avec deux alphabets d'origine étrangère : l'alphabet arabe et l'alphabet latin. Il ne sert qu'à la rédaction de textes courts : lettres à des proches, graffiti sur les arbres, sur les rochers ou les ustensiles quotidiens. L'article se concentre sur l'usage dans cet alphabet dans la pratique épistolaire, tout en faisant sa place à l'usage dans la même pratique de l'alphabet latin. Il aborde également les tentatives faites aujourd'hui pour moderniser cet alphabet.
Dominique Casajus, Everyday writing among the Tuareg. - The Tuareg have an old alphabet with characters called tafineq, a word that might refer back to the term used by the Greeks to refer to Phoenicians. This autochtonous alphabet derived from older « LibycoBerber" scripts, which are attested as far back as the middle of the second century BCE. It coexists with two alphabets of foreign origin: Arabic and Latin. It is only used to write short texts: letters to people who are close, graffiti on trees or rocks, or inscriptions on utensils. This article focuses on how this alphabet is used for letterwriting even though this activity allows for using the Latin alphabet too. Recent attempts to reform the Tuareg's alphabet are discussed. 